\title{
Service Use Patterns for Adolescents with ADHD and Comorbid Conduct Disorder
}

\author{
Damon E. Jones, PhD, E. Michael Foster, PhD, and \\ University of North Carolina-Chapel Hill, Chapel Hill, NC, USA
}

Conduct Problems Prevention Research Group

\begin{abstract}
Service use patterns and costs of youth diagnosed with attention-deficit/hyperactivity disorder (ADHD) and comorbid conduct disorder (CD) were assessed across adolescence (ages 12 through 17). Featured service sectors include mental health, school services, and the juvenile justice system. Data are provided by three cohorts from the Fast Track evaluation and are based on parent report. Diagnostic groups are identified through a structured assessment. Results show that public costs for youth with ADHD exceed $\$ 40,000$ per child on average over a 6-year period, more than doubling service expenditures for a non-ADHD group. Public costs for children with comorbid ADHD and CD double the costs of those with ADHD alone. Varying patterns by service sector, diagnosis, and across time indicate different needs for youth with different conditions and at different ages and can provide important information for prevention and treatment researchers.
\end{abstract}

\section{Introduction}

Among the many studies examining the impact of attention-deficit/hyperactivity disorder (ADHD) diagnoses on adolescents are those focusing on the rates of service utilization. Such research is important for understanding the degree to which the disorder may be affecting individuals and their families as well as the extent to which private and public resources are burdened. Much of this research examines the healthcare system-including mental health therapy as well as medication used in treatment—and study objectives are often to compare health expenditures of those with ADHD to those with other chronic conditions such as asthma $a^{1,2}$ or other behavioral diagnoses. ${ }^{3}$ Overall, considerable evidence suggests that health care expenditures for children with ADHD significantly exceed those for children not diagnosed. ${ }^{1,3-8}$ (For a thorough review of the studies examining service utilization among ADHD cases please see Matza et al. ${ }^{9}$ ).

Despite the increase in such research, few service use studies explore the variation in public costs among children with ADHD. Comorbidities amplify the link between ADHD and service use ${ }^{10}$ and raise the risk for long-term outcomes such as violence and substance abuse. ${ }^{11}$ When considering youth with ADHD, the rates for having other behavioral conditions are much higher than for the general population. For instance, while research estimates that the prevalence of conduct disorder (CD) ranges from $2 \%$ to $6 \%$ across all

(C) 2008 National Council for Community Behavioral Healthcare.

Address correspondence to Damon E. Jones, PhD, Penn State University, University Park, PA, USA. Phone: +1-814-8657177. Fax: +1-814-8630000. dejones@psu.edu.

Members of the Conduct Problems Prevention Research Group are (in alphabetical order) Karen L. Bierman, Department of Psychology, Penn State University; John D. Coie, Department of Psychology, Duke University; Kenneth A. Dodge, Center for Child and Family Policy, Duke University; Mark T. Greenberg, Department of Human Development and Family Studies, Penn State University; John E. Lochman, Department of Psychology, The University of Alabama; Robert J. McMahon, Department of

Psychology, University of Washington; and Ellen E. Pinderhughes, Department of Child Development, Tufts University. 
children, ${ }^{12}$ that rate varies between $30 \%$ and $50 \%$ for those already diagnosed with ADHD. $9,13-15$ That as many as half of all ADHD children also may have CD highlights the importance of considering how the presence of both disorders might complicate impairment and subsequent treatment strategies. Indeed, studies indicate that youth with both conditions had a higher degree of substance abuse problems than children with ADHD alone ${ }^{16}$ and are more likely to engage in criminal activity. ${ }^{17}$ Research indicates, however, that the presence of CD may have the most impact on delinquency outcomes among comorbid (ADHD-CD) children. ${ }^{18,19}$

Previous studies suffer from limitations in the methods used to examine expenditures for ADHD. First, the results are usually combined from many years of data without distinguishing at what ages expenditures occur. Such approaches exclude information on spending trends that show what service resources are most utilized at different ages and ignore how expenditures at certain ages may contribute more to total costs across a period. For instance, average expenditures at age 17 may account for 50\% of costs between ages 12 and 17. Additionally, results may be based on contrasts among sub-samples whose mean ages differ (e.g., ADHD versus asthmatic groups) whereby expenditure disparities could be partly attributable to age differences of diagnostic groups.

Second, most studies identify samples through observation of the disorder in medical records, e.g., ADHD status in the study is based on provider diagnosis or insurance claim coding from service visits. Such diagnoses may reflect, as well as cause, medical expenditures, i.e., children who receive more services may be more likely to have their condition identified as related to ADHD. Determining ADHD status this way also raises key issues about representativeness since these sampling strategies exclude individuals not seeking treatment during the study period or who happened not to be identified with the condition by the provider. Excluding ADHD cases who have not been detected in medical records could skew outcomes in both directions. For example, expenditures will be underestimated for children who have not sought healthcare in the observation period but are requiring many school or criminal justice system services. On the other hand, average expenditures could be overestimated by excluding youth who genuinely have ADHD but do not require as many services (i.e., genuine ADHD subjects are falsely included in the nonADHD group due to their lack of medical history).

Additionally, certain areas of social expenditures are underexamined. Expenditures for those with ADHD in the juvenile justice system have been virtually unexplored, and costs of ADHD related to the school system are limited to overall expenditures without any examination of individual trends in cost. By ignoring juvenile justice and education, prior studies omit a considerable segment of service expenditures.

This retrospective study considers the public costs of ADHD while improving on prior research. First, expenditures are examined by year for a 6 -year period covering late middle school and early high school ages. This approach allows exploration of trends in expenditures during a period when youths require a wide range of services, contrasting development among children with diagnostic distinctions. The method for determining diagnoses also improves on prior research. Subjects were grouped based on a clinical assessment of behavioral health symptoms, so ADHD status was determined independently of their history with the service sectors. Third, in addition to examining medical and mental health expenditures, school system and juvenile justice system expenditures also are a focus in this research. Finally, the impact of co-occurring $\mathrm{CD}$ is assessed given its high comorbidity rate with ADHD and its importance in service expenditures research. The primary focus of this paper is on the relationship between ADHD diagnosis and services use, and so the results first will be presented ignoring the possibility of co-occurrence of $\mathrm{CD}$ (i.e., 
where youth with CD but not ADHD would be included in the non-ADHD group). The second approach will acknowledge the possible co-occurrence of a CD diagnosis, dividing the sample into four groups: those with ADHD alone, those with $\mathrm{CD}$ alone, those with both conditions, or those with neither conditions. Both perspectives (comparing two groups and comparing four groups) are presented in order to help us understand how results might differ depending on whether or not such important co-morbidities are considered.

\section{Methods}

The data for these analyses were collected as part of the Fast Track project, ${ }^{20}$ a multi-cohort, multi-site longitudinal study of children who are at risk for externalizing behavior problems. The sample $(N=650)$ excludes children receiving intervention services and instead includes those who were (a) screened into the high-risk comparison group, and (b) a "normative" sample recruited to allow for examination of trends in a more representative group of youth. Children were recruited from the population of kindergarteners in 54 schools $(9,594$ subjects) spread across three urban sites (Durham, NC; Nashville, TN; Seattle, WA, USA) and one rural (central Pennsylvania). Children in the high-risk group were identified using a two-stage screening process where those scoring in the top $40 \%$ within cohort and site on a teacher assessment of behavior problems were then screened using a parent measure of home behavior problems. ${ }^{20}$ Children were selected for inclusion into the study based on this screen score, moving from the highest score downward until desired sample sizes were reached within sites, cohorts, and conditions. Children for the lower risk/normative sample were recruited from those not identified as at-risk for behavior problems based on teacher and parent combined assessment. Across the four sites, approximately 50\% of children are African-American, $41 \%$ involved subjects from a single-parent household (at the start of measurement in this study), and the overall SES was between lower and lower middle class. ${ }^{21}$ The recruitment process was repeated for the next 2 years to create three cohorts. Basic characteristics of the sample (gender, race, and risk status) by site are provided in Table 1.

Because of the oversampling of at-risk children, sampling weights were calculated based on the probability that a child was recruited for the study. These weights were used to adjust outcomes presented below to more accurately represent the original populations eligible for the study in the four communities. ${ }^{*}$ The result is a sample that, when weighted, is representative of the population but yet contains enough high-risk individuals to estimate public expenditures relatively precisely. ${ }^{22}$

Information on service use was derived from interviews with participating families and from administrative data. The latter procedure involved a review of school records every summer, which provided information on whether a youth repeated a grade or received special education in each year. ${ }^{23}$ Information on the use of health and mental health services as well as juvenile justice involvement was provided by parents/primary caregivers in the Service Assessment for Children and Adolescents (SACA) ${ }^{24}$ an instrument shown to have good reliability and validity characteristics for similar populations to the Fast Track sample..$^{25,26}$ For the purposes of this study, the reported frequency of service visits/number of service days in the past year was used as the measure of service usage for assessed service sectors. Additionally, caregivers provided annual information as to whether or not their child required medications for emotional/behavior problems in an annual assessment of "life changes." ${ }^{27}$ All of this information was first collected when youth were in 6th grade and

\footnotetext{
* Racial breakdown varies substantially across sites as sample composition reflects the characteristics of the representative high-risk youth in the respective communities. The confounding of site with race is addressed in statistical models using covariates. Any further focus on varying service patterns across racial and/or regional differences is beyond the scope of this study.
} 
continued through high school. (Results are presented in terms of approximate age since children were not necessarily in the same grade because of retention.) Measures were administered using identical data collection procedures and timing for the three cohorts except where noted below.

Missing data rates were typical for this type of longitudinal sample, ranging from approximately $20 \%$ to $30 \%$ (depending on measure) by year 12 (grade 11) of the project. A different assessment schedule of the SACA in earlier years further affected sample size; namely, cohort 1 did not receive the SACA at age 13 leading to a uniquely higher missing data rate for that measure/year (i.e., almost $2 / 3$ of the sample were missing for the SACA at age 13 for this cohort). To address any concerns for non-randomly missing data across measures, full sample parameter estimates for the 6-year time period were achieved through multiple imputations (MI). The use of MI routines to accommodate missing data has been shown to enable accurate analytic outcomes while not requiring missingness patterns to follow a completely random process. ${ }^{28}$ Research has demonstrated that MI methods for accommodating missing data in analyses are efficient even when missing data rates are high for some variables. ${ }^{29}$ Appendix A provides additional information on the MI routines as well as the sample sizes for the various measures.

At age 12 and age $15,{ }^{*}$ children were assessed for ADHD using the Diagnostic Interview Schedule for Children (DISC). ${ }^{30}$ Parents were asked whether their children demonstrated symptoms related to the disorder during the past year. Children exhibiting six or more of these symptoms on either the ADHD-Inattention or ADHD-Hyperactivity/Impulsivity scales were diagnosed as having ADHD. Criteria for this diagnosis are set by the DISC scoring program. Fifty-six $(9 \%)^{\dagger}$ of the children received a diagnosis of ADHD at age 12, and 48 $(8.5 \%)$ of the children at age 15 received the diagnosis. For the purposes of this study, a child was considered as having ADHD based on a positive diagnosis at either age 12 or age 15. Using that criterion, 84 (15.3\%) children were labeled ADHD. This percentage when weighted using sampling weights is $6.4 \%$, which is within the range of the typical prevalence reported for the disorder. ${ }^{31}$ Males were more likely to be diagnosed in either year (20.4\% males versus $8.5 \%$ females), and children initially identified as high risk in the Fast Track sample were almost seven times as likely to have a diagnosis in either year $(24.0 \%$ versus 3.5\% for the "non-high-risk" children).

To examine the possible impact of comorbid CD on service expenditures, the sample was further divided into four groups: children diagnosed with both ADHD and CD, children diagnosed with ADHD alone, children diagnosed with $\mathrm{CD}$ alone, and children diagnosed with neither condition. The DISC also was used to determine CD diagnosis at the same ages (again, diagnoses were based on DISC scoring criteria; diagnosis of Conduct Disorder was based on the presence of three or more symptoms for that construct). Within this group breakdown, 23 (3.5\%) subjects were shown to have both ADHD and CD, 36 (5.5\%) had CD only, $61(9.4 \%)$ had ADHD only, and $530(81.5 \%)$ had neither diagnosis.

Featured outcomes cover several categories, combined from specific service sectors. The categories (and the service sectors included) are as follows: inpatient mental health expenditures (psychiatric hospital, residential treatment center, group home, foster care); outpatient mental health expenditures (drug and alcohol clinic, day treatment center, mental health center, in-home provider, individual counselor/therapist); general health (emergency

\footnotetext{
* The Fast Track project assessed behavioral conditions at age 9, age 12, and age 15. The latter two measurement periods were used to assess ADHD and CD since they are proximal to the period of adolescence when service trends are examined, and also provide diagnoses based on behavior across a full year (the earlier assessment was only based on a 6-month period).

$\dagger$ These numbers are based on pre-imputed data. All figures in this section are unweighted $N$ s and percentages (except where noted). Weights were used in all cost calculations and significance tests presented in the "Results" section.
} 
department, family doctor, general hospital); juvenile justice (detention center, arrest expenditures); and school (school counseling, special education, grade retention). Medication expenditures were the only single item category.

Per-unit expenditures were calculated as the price that a unit of government pays for the service or treatment. Most expenditures used in calculations come from follow-up examination of service use (record reviews) within this sample. There were a few exceptions where more reliable estimates were attained from prior research: costs of special education, ${ }^{32}$ grade retainment, ${ }^{33}$ juvenile justice system services,,${ }^{34}$ and medication. ${ }^{35}$ All figures were converted to 2000 dollars ${ }^{*}$ using the Consumer Price Index. More details on per-unit costs are provided in Appendix B. Service use amounts are not represented for age 12 in the justice system sector and age 13 in the mental health sector due to a combination of low response rates and/or too much missing data to do accurate imputations.

\section{Results}

Service use amounts are presented as overall expenditures for each year and across designated diagnostic groups. Figure 1 provides time trends for the average expenditures contrasting youth with and without ADHD (here, presence of other conditions such as CD are ignored). Figure 2 provides time trends for the four diagnostic groups defined above, representing comorbidity of ADHD with CD. Variation in expenditures between groups was assessed using separate regression analyses by age. Separate models were used for the assessment of significant change across time. Cost outcomes were logged in regressions to lessen the impact of extreme values. Dummy variables for project site, gender, and race (whether African-American) were entered as covariates in all statistical models. Significance tests were performed using MI routines and $p$ values are presented here; significant $p$ values are given in terms of criterion level $(0.05,0.01,0.005)$. Results are presented corresponding to the three research foci discussed in the "Introduction".

\section{Results focusing on age}

Figures 1 and 2 show how, to varying degrees, service expenditures change across adolescence. In most cases, service sector totals increase. This is not always the casemedication use is stable or decreases slightly in this age period. Costs for the mental health and justice system sectors increased significantly $(p<0.01)$. Change in total service expenditures is not significant due to the inconsistency of change across service sectors and high variation within the sample.

\section{Results contrasting diagnostic groups}

Figure 1 displays mean costs for children with and without ADHD. Total costs exceed $\$ 9,300$ on average for the ADHD group by age 17 , approximately 2.4 times the amount for the children without ADHD for that age. Total costs were significantly greater for youth with ADHD at each age (levels range from 0.05 at ages $12-13$ to 0.005 at later ages). The jump in average total costs at age 14 reflects increases in other service sectors, primarily school and mental health. Disparity between ADHD grouping for medication use occurs across ages ( $p<0.005$ for ages $12-14 ; p<0.05$ for ages 15-16; non-significant at age 17). While mean trends for costs within the justice system between ADHD and non-ADHD groups appear to diverge by age 17 (Fig. 1), such differences were not statistically significant.

\footnotetext{
* The year 2000 was used as it represents a central timepoint between the earliest data collection for these outcomes (1997 for cohort 1) and the latest (2004 for cohort 3 ).
} 
Figure 2 presents service expenditures considering potential comorbidity of ADHD with $\mathrm{CD}$. Expenditures for the group with neither diagnosis stay relatively stable in all service sectors. In contrast, the three diagnosed groups increase at varying levels. Significance tests indicate high variation between groups on total costs at all ages $(p<0.005)$. Disparities in total costs are most evident in later years where comorbid and $C D$ youth have higher expenditures. Significant between group variation also occurs for total mental health costs ( $p<0.005$ at all ages), behavioral medications [ $p<0.005$ for all years except for age 16 $(p<0.05)]$, school expenses ( $p<0.005$ for ages 14, 16, and 17; $p<0.05$ for age 15), and for justice system expenditures in later years $(p<0.05$ for age $15 ; p<0.005$ for ages 16 and 17).

Variation in proportional expenditures by comorbidity group is shown in Figure 3 for the highest expenditure service sectors (total mental health is divided into inpatient and outpatient). Despite differences in overall expenditures, the neither-diagnosis and ADHDonly groups have similar patterns of proportional spending with the exception of inpatient mental health expenditures (higher proportion for the latter group). Inpatient mental health services play a greater role for comorbid youth than for youth with ADHD alone (34.6\% versus $22.4 \%$ ); in contrast, the $\mathrm{CD}$ group spends approximately a quarter of their total expenditures in the justice system sector. The presentation of both overall levels and proportional expenditures is important to this figure. For instance, while ADHD and neitherdiagnosis groups have roughly the same proportional expenditures for school services (a little more than half of total dollars), the ADHD group still requires more than twice as many resources in that sector.

\section{Results focusing on juvenile justice and school system sectors}

Both school and justice system service needs increase across adolescence to reach highest levels by age 17. Contrasts between ADHD status for justice system expenditures are nonsignificant across ages ${ }^{*}$ where $\mathrm{CD}$-only diagnosed youth belong to the non-ADHD group. The significant group variation for justice system expenditures among comorbid diagnostic groups (noted above) is explained by $\mathrm{CD}$ and comorbid groups having much higher service expenditures at ages 15-17. Notably, justice system service needs are similarly low for all groups at younger ages. For the school services sector, significant disparity is found between groups from ages 14 through 17 regardless of whether considering comorbidity. In both cases, the non-diagnosed group is significantly lower in school service needs for the older ages.

\section{Discussion}

The results from these analyses show how youth with varying diagnoses differ in their service expenditures to a substantial degree as reported by parents. Over a 6-year period, service costs for the ADHD group exceeded the non-ADHD group by $\$ 25,000$ per child on average. For youth classified as both $\mathrm{ADHD}$ and $\mathrm{CD}$, the average service expenditures were greater than $\$ 80,000$ over 6 years - more than twice as high as youth with ADHD only-and six times the amount for a child with neither disorder. Data on annual service expenditures provide useful information for policy makers and prevention researchers, indicating the necessary service resources required for different ages and different conditions. Researchers can use information on the timing of the most intense service needs to help understand the impact of such conditions in adolescence and, in extension, when intervention may be most useful. While the need for services may not be exhaustively explained by a disorder(s), the association is important.

\footnotetext{
${ }^{*}$ Differences are marginally significant at age $17, p<0.10$.
} 
Expenditures vary not only in level but in composition as well. While not apparent in the diagnosis level figures presented here, such variation exists within categories. For example, supplemental exploration of the data showed that, averaging across years, school service costs and juvenile justice costs are positively correlated among ADHD youth, a trend not seen among $\mathrm{CD}$ or comorbid youth. These figures suggest that among those with an ADHD condition (but not $\mathrm{CD}$ ), the need for assistance at school might reflect general disciplinary problems that are not detected through a formal diagnosis. Other relationships that one might anticipate are not apparent. For instance, one might expect outpatient mental health service costs to be negatively correlated with justice system costs. Simple correlations of average costs did not confirm such patterns. The lack of a relationship could be a function of contrasting situations, e.g., in some cases mental health services may prevent juvenile delinquency; in other cases, court sentencing may instigate mental health services. Regardless of these facts, youth are (hopefully) not assigned randomly to services in the real world, so these relationships will reflect a range of other factors, such as severity. Future research could address the underlying causal relationships among service delivery from multiple sectors.

Several limitations apply to these findings. The results are based on cost averages across individuals. As is typical in research on services needs, expenditures can be concentrated on a minority of children receiving intensive services. Conditional averages (i.e., the average expenditures among those children identified as needing services) would better reflect the costs for children with behavioral problems but are not as helpful in estimating population averages which incorporate those who do not require services. Also, children were classified as impaired if the parent DISC indicated a positive diagnosis at either age 12 or 15 . This approach is not atypical but assumes that youth with a diagnosis at one of the two assessments has a similar condition to youth with a diagnosis at both assessments. Finally, estimates that were derived from dichotomous items (whether or not received assistance)in this study this applies to medication use and special education-under-represent the true variation in the service intensity and expense seen in real settings.

Variation in expenditures across youth of different ages may reflect concurrent policy changes. Such factors are not likely to lead to substantial differences between cohorts separated by only 2 years. However, preliminary statistical models assessed the impact of cohort status in order to ensure that there were no differences between sub-samples. These covariates were removed from the final statistical models when no differences between cohorts were observed. This finding was expected given the nature of the cohorts from the Fast Track sample. Regardless of this finding, future research from any study should consider the possibility of variation in health, school, and justice system service trends simply as a function of historical timing.

Clearly, the costs presented above are a subset of the overall social costs involved. For example, the school costs captured above are substantial but ignore the effects of school failure on a youth's long-term earnings or even the immediate effects of classroom disruption on other children. Juvenile justice costs do not begin to capture the impact of crime on victims. Additionally, since the SACA mostly assesses services related to emotional/behavioral needs, much more likely is spent in the general health sector than is detected with these data. The amounts shown represent an important portion of the expenditures related to these conditions. In general, costs expended in the health sector often imply costs elsewhere, not just in the education and juvenile justice sectors, but also in terms of lost employment income, higher rates of substance abuse, and quality of life costs such as higher divorce rates. ${ }^{36}$ 
Finally, our findings do not incorporate the benefits of service use. Presumably, such services are deemed necessary in order to improve future outcomes for youth with ADHD and/or CD (even in the case of juvenile justice services). But the effectiveness of these observed service levels is unknown. The intensity and expense of certain services may reflect more the degree of impairment than the degree of future improvement that might be expected. Investigating the effectiveness of services is a challenge when using observational data since it is hard to determine important causal relationships, especially among youth who are served in so many different sectors (investigators would have to consider the many important interactions between multiple service types and among important subject-level characteristics). Typically, the specifics of the service delivery (e.g., the actual treatment strategy or approach used in an outpatient mental health therapy) are not detailed in the data, as is the case with the data used here. Such information is necessary to distinguish why certain services work and others are not effective. Another challenge is the need to follow subjects into adulthood in order to understand the long-term impact of service receipt. Despite the importance of understanding the amount of necessary services for impacted youth, the efficacy of treatments for ADHD and/or CD will continue to mostly rely on standard randomized control studies.

\section{Implications for behavioral health}

Assessment of comorbidity is clearly important when linking diagnoses to services use. Certainly, evaluation of a single diagnosis (ADHD versus non-ADHD) has limited utility since those not diagnosed may actually have other important conditions that may be worth distinguishing. This paper examines perhaps the most important comorbid condition for ADHD youth-the diagnosis of CD. Results show that youth with both conditions may have different patterns of service needs than those with a single diagnosis. This pattern may be most obvious for the justice system sector: service needs for comorbid and CD children are similarly high whereas ADHD youth more resemble the neither-diagnosis group. This finding is consistent with previous research indicating that juvenile justice service needs are driven by the presence of $\mathrm{CD}^{18,19}$ but possibly exacerbated by comorbidities. Another important observation is the substantially higher service needs for comorbid youth in the mental health service sector during later adolescence (Fig. 2). This pattern is further demonstrated by the higher proportion of inpatient mental health costs for youth with both ADHD and CD than for those with either condition alone (Fig. 3). Such a difference might be explained by the greater need among CD or comorbid cases for more expensive psychotherapy services (whereas less expensive medication treatment might be sufficient in treating many with ADHD alone). Cormorbid conditions beyond $\mathrm{CD}$ are left for other research.

In general, the variation in expenditures across conditions might affect decisions for how to target preventive resources. For instance, the results above imply that treatment to prevent aggressive behavior may be most important for those that are diagnosed with $\mathrm{CD}$ regardless of their ADHD status. When considering ADHD alone, research indicates that it is important to acknowledge the subclassifications-distinguishing between ADHD with an aggressive subtype versus ADHD with an anxiety subtype-when determining preventive strategies. ${ }^{37}$ But this especially may be the case if ADHD children actually have a comorbid CD diagnosis. Information on the degree of need among comorbid youth might also be helpful in directing necessary intervention to those already impaired. This possibility could include introducing a combination of medications that are most appropriate for treating adolescents with ADHD and CD. ${ }^{38}$ Specific targeting may be most helpful in reaching the children who are worse off (most affected by their disorder or by multiple disorders). Of course, any successful preventive endeavor is dependent on early identification of children with such conditions or at risk for such conditions. 
The relationships among the disorders and treatment should be considered in terms of timing as well. Research indicates that while children with both conditions may differ genetically from children with ADHD alone, environmental factors influence the onset of CD. ${ }^{39}$ Perhaps what distinguishes the comorbid youth from those with ADHD alone is early treatment that reduces the impact of the child's surroundings. That treatment may have deterred processes from starting (and evolving) that reinforce problem behaviors.

There are logical extentions to this research that could be pursued in order to further understand the needs of these youth. The window of observation might be expanded both in terms of time and domain, with efforts to clarify spending trends related to ADHD and comorbid conditions from childhood through early adulthood while incorporating information from other sources (such as medical records) and expanding the service sectors examined (e.g., investigating further the impact on general health service needs or alcohol and drug abuse treatment). Further research also might examine how trends vary among those with ADHD and/or CD. A focus here could distinguish between those diagnosed with ADHD primarily due to inattention symptoms versus those diagnosed primarily based on hyperactive symptoms. As with many issues in the behavioral sciences, the better the understanding of the underlying conditions and their consequences (such as need for intense and expensive services), the greater the hope for initiating effective timely intervention or preventive efforts.

\section{Acknowledgments}

This work was supported by National Institute of Mental Health (NIMH) grants R18 MH48043, R18 MH50951, R18 MH50952, and R18 MH50953. The Center for Substance Abuse Prevention and the National Institute on Drug Abuse also have provided support for Fast Track through a memorandum of agreement with the NIMH. This work was also supported in part by the Department of Education grant S184U30002 and NIMH grants K05MH00797 and K05MH01027. The economic analysis of the Fast Track project is supported through R01MH62988. The content is solely the responsibility of the authors and does not necessarily represent the official views of the National Institute On Drug Abuse or the National Institutes of Health.

\section{Appendix A: Using multiple imputation to replace missing values}

Missing data occurred in the Fast Track sample because of typical study attrition as well as because of different assessment schedules in earlier years (e.g., cohort 1 did not receive the SACA in year 8). Multiple imputation (MI) enables researchers to perform complete case analyses while acknowledging the variation inherent to accommodating missing data. Simulations have demonstrated that MI routines lead to valid analytic results given the assumption that data are conditionally missing-at-random (i.e., missingness is explained by non-missing covariates in the imputation model). Further details on the computer-intensive estimation processes involved in MI can be found elsewhere. ${ }^{40}$

Table 2 shows the actual total sample size for each year and source of data.

Outcomes were averaged across ten multiply imputed datasets, and variations in the imputed values were factored into significance tests. Imputation models included all of the service outcomes for all years, as well as diagnosis variables (conduct disorder, kindergarten risk status) and key demographic variables (race, gender, cohort, study site). Models were run using IVEWare, ${ }^{41}$ an imputation program that provides flexibility in estimating models with Poisson-distributed count variables.

\section{Appendix B: Service expenditures}

Per-unit expenditures were derived from two sources: estimates from previous research and estimates from record reviews of services being delivered to the Fast Track sample (more 
below). Table 3 provides the source for each cost estimate used. Total expenditures per child were calculated by multiplying the per-unit expenditure in Table 3 by the service amount number provided by the parent/caregiver.

An agency review was instigated in year 9 of the Fast Track project (when most youth were in 8th grade) to obtain more detailed information on service delivery within the sample. Parents who reported service use in the past year were asked for written permission to follow up with facilities they could identify. Information recorded in these reviews included costs of services delivered. Estimates for expenditures per service from this supplemental data were used for sectors that did not have reliable estimates available from the literature (noted in Table 3). The following clarifies the identification of per-unit costs from the literature:

\section{School services}

School expenditures were comprised primarily of special education and grade retention. Special education expenditures were taken from Forness and Kavale 32 (pp. 24-28) using data from 19 states. The overall average estimate for the excess cost of special education services was used $(\$ 5,435)$ as provided by these authors.

National Center for Education Statistics estimated the per pupil grade retention expenditure as $\$ 6,508$ in school year $1998-1999 .{ }^{33}$ This expenditure included instructional services, support services, and non-instructional services.

\section{Juvenile justice services}

Juvenile justice arrest processing expenditure estimates are available in the literature based on the type of crime. Given the typical crime committed by this population, arrest expenditures were based on robbery-related offenses (in contrast to higher costs of arrest for such offenses as murder and rape). ${ }^{34}$ The average cost in 1987 dollars $(\$ 1,125)$ was adjusted to 2000 dollars for computations.

\section{Medication}

Using the MEPS, Zuvekas ${ }^{35}$ estimated spending for psychotropic drugs (antidepressants, antianxiety, antipsychotics, stimulants, and sedative hypnotics). Among youth aged from 6 to 17 who received these medications, related expenditures averaged $\$ 318$ per person in 1996.

\section{References}

1. Chan E, Zhan C, Homer CJ. Health care use and costs for children with attention-deficit/ hyperactivity disorder. Archives of Pediatrics \& Adolescent Medicine. 2002 May; 156(5):504-511. [PubMed: 11980558]

2. Kelleher, KJ. Use of services and costs for youth with ADHD and related conditions. In: Jensen, PS.; Cooper, JR., editors. Attention Deficit Hyperactivity Disorder. Kingston, NJ: Civic Research Institute; 2002. p. 27-21-27-12.

3. Mandell DS, Guevara JP, Rostain AL, et al. Medical expenditures among children with psychiatric disorders in a Medicaid population. Psychiatric Services. 2003; 54(4):465-467. [PubMed: 12663833]

4. Birnbaum HG, Kessler K, Lowe S, et al. Costs of attention deficit-hyperactivity disorder (ADHD) in the US: excess costs of persons with ADHD and their family members in 2000. Current Medical Research Opinion. 2005; 21:195-206. 
5. Guevara J, Lozano P, Wickizer T, et al. Utilization and cost of health care services for children with attention-deficit/hyperactivity disorder. Pediatrics. 2001; 108(1):71-79. [PubMed: 11433056]

6. Leibson CL, Katusic SK, Barbaresi WJ, et al. Use and costs of medical care for children and adolescents with and without attention-deficit/hyperactivity disorder. Journal of the American Medical Association. 2001 Jan 3; 285(1):60-66. [PubMed: 11150110]

7. Swensen AR, Birnbaum HG, Secnik K, et al. Attention-deficit/hyperactivity disorder: increased costs for patients and their families. Journal of the American Academy of Child \& Adolescent Psychiatry. 2003 Dec; 42(12):1415-1423. [PubMed: 14627876]

8. Szatmari P, Offord DR, Boyle MH. Correlates, associated impairments and patterns of service utilization of children with attention deficit disorder: findings from the Ontario child health study. Journal of Child Psychology and Psychiatry. 1989; 30(2):205-217. [PubMed: 2468679]

9. Matza LS, Paramore C, Prasad M. A review of the economic burden of ADHD. Cost Effectiveness and Resource Allocation. 2005; 3:5. [PubMed: 15946385]

10. Burd L, Klug M, Coumbe M, et al. The attention-deficit hyperactivity disorder paradox: 2 . Phenotypic variability in prevalence and cost of comorbidity. Journal of Child Neurology. 2003; 18:653-660. [PubMed: 14572145]

11. Arseneault L, Moffitt TE, Caspi A, et al. Mental disorders and violence in a total birth cohort: results of the Dunedin Study. Archives of General Psychiatry. 2000; 75(10):979-986. [PubMed: 11015816]

12. Hinshaw, SP.; Lee, S., editors. Conduct and oppositional defiant disorders. 2. New York: Guilford Press; 2003. Mash, RABEJ., editor. Child psychopathology.

13. Kuhne M, Schachar RJ, Tannock R. Impact of comorbid oppositional or conduct problems on attention-deficit hyperactivity disorder. Journal of American Academic Child Adolescent Psychiatry. 1997; 36(12):1715-1725.

14. Disney ER, Elkins IJ, McGue M, et al. Effects of ADHD, conduct disorder, and gender on substance use and abuse in adolescence. American Journal of Psychiatry. 1999 Oct; 156(10):15151521. [PubMed: 10518160]

15. Daley CE, Griffin H, Onwuegbuzie AJ. Prevalence and identification of attention-deficit hyperactivity disorder in a mid-southern state. Research in the Schools. 1997; 4(2):49-56. Fal.

16. Barkley RA, Fischer M, Smallish L, et al. Does the treatment of attention-deficit/hyperactivity disorder with stimulants contribute to drug use/abuse? A 13-year prospective study. Pediatrics. 2003 Jan; 111(1):97-109. [PubMed: 12509561]

17. Babinski LM, Hartsough CS, Lambert NM. Childhood conduct problems, hyperactivityimpulsivity, and inattention as predictors of adult criminal activity. Journal of Child Psychology and Psychiatry. 1999 Apr; 40(3):347-355. [PubMed: 10190336]

18. Mannuzza S, Klein RG, Konig PH, et al. Hyperactive boys almost grown up. IV. Criminality and its relationship to psychiatric status. Archives of General Psychiatry. 1989 Dec; 46(12):10731079. [PubMed: 2589922]

19. Satterfield JH, Schell A. A prospective study of hyperactive boys with conduct problems and normal boys: adolescent and adult criminality. Journal of the American Academy of Child \& Adolescent Psychiatry. 1997 Dec; 36(12):1726-1735. [PubMed: 9401334]

20. Conduct Problems Prevention Research Group. A developmental and clinical model for the prevention of conduct disorders: the Fast Track Program. Development and Psychopathology. 1992; 4:509-527.

21. Hollingshead, AB. Four factor index of social status: an unpublished manuscript. New Haven, CT: Yale University; 1975.

22. Thompson, SK. Sampling. New York: Wiley; 1992.

23. Walker, HM.; Block-Pedego, A.; Todis, B.; Severson, H. School Archival Records Search (SARS): User's guide and technical manual. Longmont, CO: Sopris West; 1991.

24. Stiffman AR, Horwitz SM, Hoagwood K, et al. The Service Assessment for Children and Adoldescents (SACA): adult and child reports. Journal of the American Academy of Child and Adolescent Psychiatry. 2000; 39(8):1032-1039. [PubMed: 10939232] 
25. Hoagwood K, Horwitz SM, Stiffman AR, et al. Concordance between parent reports of children's mental health services and services records: the Services Assessment for Children and Adolescents (SACA). Journal of Child and Family Services. 2000; 9:315-331.

26. Horwitz SM, Hoagwood K, Stiffman AR, et al. Measuring youth's use of mental health services: reliability of the Services Assessment for Children and Adolescents (SACA). Psychiatric Services. 2001; 52:1088-1094. [PubMed: 11474056]

27. Dodge KA, Bates JE, Pettit GS. Mechanisms in the cycle of violence. Science. 1990; 250:16781683. [PubMed: 2270481]

28. Little, RJA.; Rubin, DB. Statistical analysis with missing data. 2. Hoboken, New Jersey: Wiley; 2002.

29. Schafer JL, Olsen MK. Multiple imputation for multivariate missing-data problems. Multivariate Behavioral Research. 1998; 33(4):545-571.

30. Shaffer D, Fisher P, Christopher PL, et al. NIMH diagnostic interview schedule for children version IV (NIMH DISC-IV: description, differences from previous versions, and reliability of some common diagnosis. Journal of American Academy of Child \& Adolescent Psychiatry. 2000; 39(1):28-38.

31. Wolraich, ML. Current assessment and treatment practices in ADHD. In: Jensen, PS.; Cooper, JR., editors. Attention Deficit Hyperactivity Disorder. Kingston, NJ: Civic Research Institute; 2002. p. 23-21.

32. Forness, SR.; Kavale, KA. Impact of ADHD on school systems. In: Jensen, PS.; Cooper, JR., editors. Attention Deficit Hyperactivity Disorder. Kingston, NJ: Civic Research Institute; 2002. p. 24-21.thru 24-30

33. National Center for Education Statistics. Statistics in Brief: Revenues and Expenditures for Public Elementry and Secondary Education: School Year 1998-1999. U S Department of Education. 2001 NCES 2001-321.

34. Cohen, MA.; Miller, TR.; Rossman, SJ. The costs and consequences of violent behavior in the United States. In: Reiss, AJ., Jr; Rother, JA., editors. Understanding and Preventing Violence: Consequences and Control of Violence. Vol. 4. Washington, DC: National Academy Press; 1994. p. 67-166.

35. Zuvekas S. Trends in mental health services use and spending, 1987-1996. Health Affairs. 2001; 20(2):214-224. [PubMed: 11260946]

36. Warner, J.; Biederman, J. ADHD costs adults $\$ 77$ billion in lost income. Available at: http:// my.webmd.com/content/article/94/102557.htm

37. Jensen PS, Martin D, Cantwell DP. Comorbidity in ADHD: implications for research, practice, and DSM-V. Journal of the American Academy of Child \& Adolescent Psychiatry. 1997 Aug; 36(8): 1065-1079. [PubMed: 9256586]

38. Connor DF, Barkley RA, Davis HT. A pilot study of methyiphenidate, clonidine, or the combination in ADHD comorbid with aggressive oppositional defiant or conduct disorder. Clinical Pediatrics. 2000; 39(1):15-25. [PubMed: 10660814]

39. Thapar A, Tharrington R, McGuffin P. Examining the comorbidity of ADHD-related behaviours and conduct problems using a twin study design. British Journal of Psychiatry. 2001; 179:224 229. [PubMed: 11532799]

40. Schafer, JL. Analysis of incomplete multivariate data. London: Chapman and Hall; 1997.

41. Raghunathan, TE.; Solenberger, PW.; Van Hoewyk, J. IVEware: Imputation and Variance Estimation Software User Guide. Survey Methodology Program, Survey Research Center, Institute for Social Research, University of Michigan; 2002. 

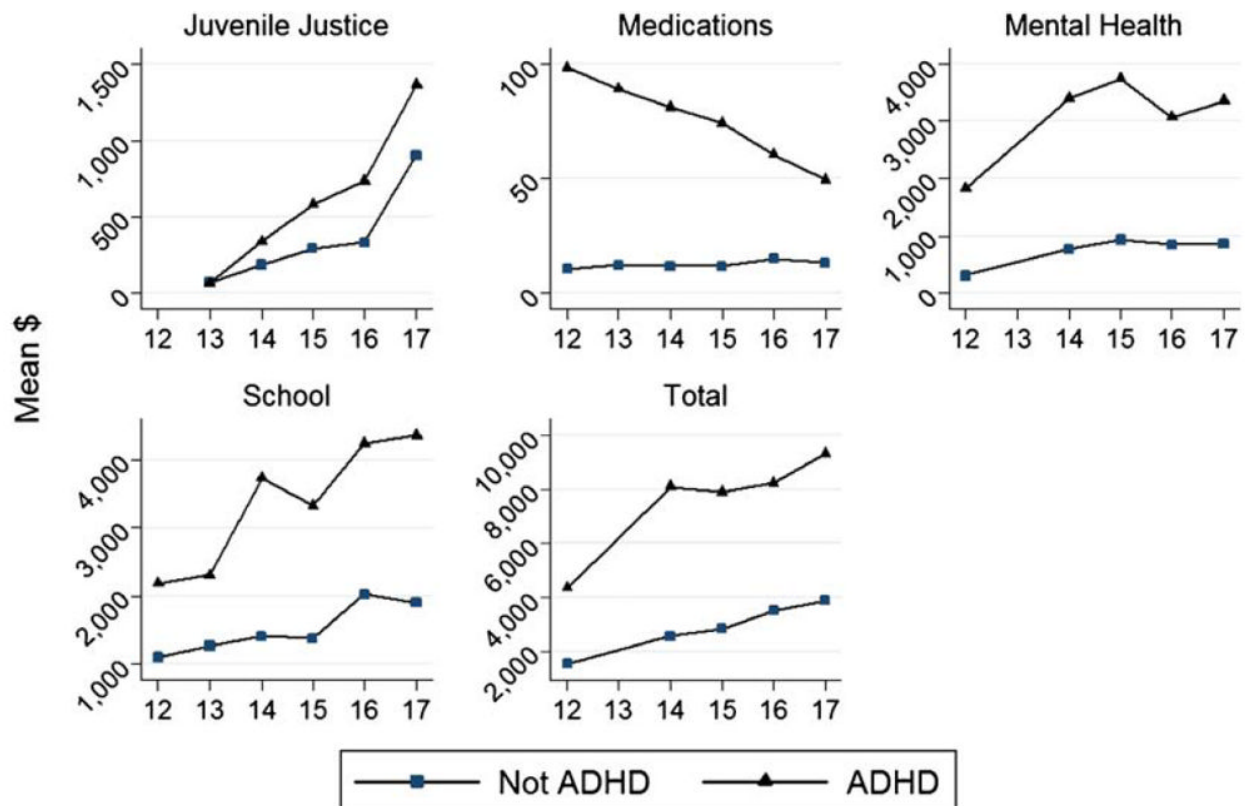

Figure 1.

Mean costs by diagnosis status across age 


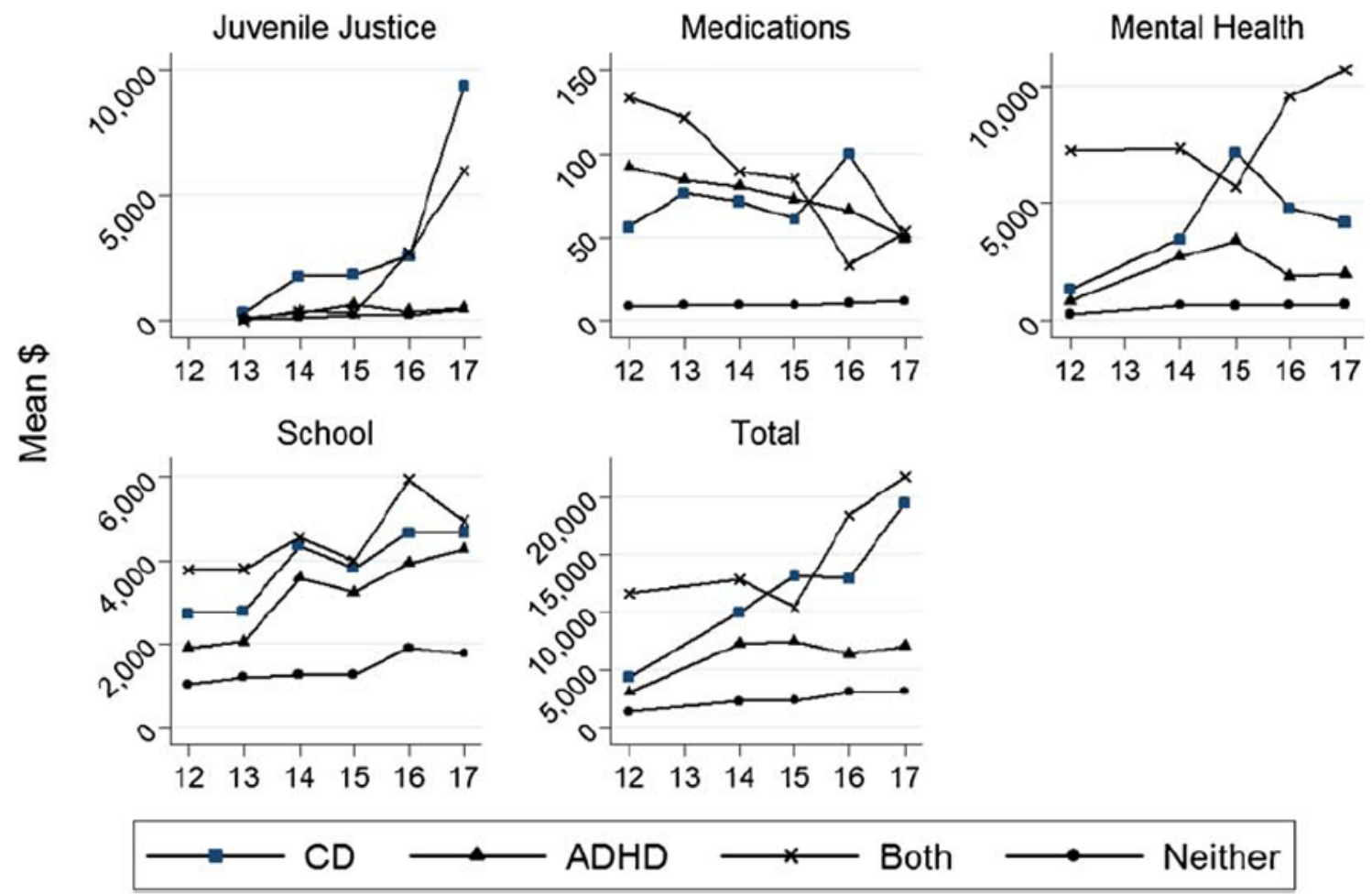

Figure 2.

Mean costs by diagnosis group status across age 


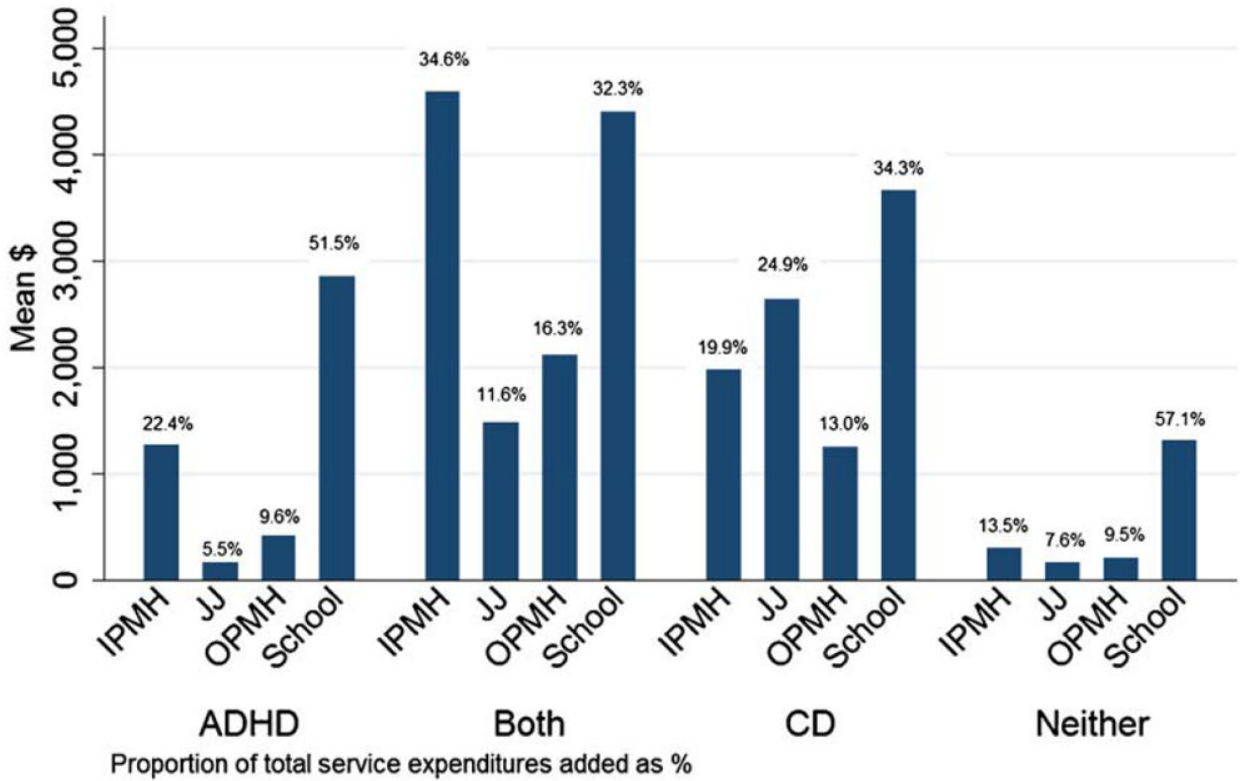

Figure 3.

Mean costs per service sector (across ages). IPMH Inpatient mental health, $J J$ juvenile justice, $O P M H$ outpatient mental health 
Table 1

Sample characteristics by site

\begin{tabular}{lrrrr}
\hline & Durham (\%) & Nashville (\%) & Central PA (\%) & Seattle (\%) \\
\hline Race & & & & \\
$\quad$ Caucasian & 8.9 & 43.0 & 98.0 & 50.3 \\
African-American & 91.1 & 57.0 & 2.0 & 34.9 \\
Other & 0.0 & 0.0 & 0.0 & 14.8 \\
Gender & & & & \\
Female & 37.8 & 45.0 & 44.8 & 40.3 \\
$\quad$ Male & 62.2 & 55.0 & 55.2 & 59.7 \\
Risk status for behavior problems & & & & \\
Higher risk & 56.7 & 62.4 & 56.4 & 59.1 \\
$\quad$ Lower risk & 43.3 & 37.6 & 43.6 & 40.9 \\
\hline
\end{tabular}


Table 2

Sample sizes providing data ( $N=650$ for entire sample)

\begin{tabular}{lcccl}
\hline & Medications & School records & SACA data & Cohorts represented \\
\hline Year 7 (age 12) & 621 & 649 & 611 & All \\
Year 8 & 603 & 638 & 221 & C2,C3 (SACA); All (others) \\
Year 9 & 580 & 626 & 578 & All \\
Year 10 & 583 & 597 & 585 & All \\
Year 11 & 558 & 542 & 557 & All \\
Year 12 (age 17) & 533 & 467 & 531 & All \\
\hline
\end{tabular}


Table 3

Cost estimates for per-unit services

\begin{tabular}{|c|c|c|}
\hline & Cost/day (\$) & Cost estimate source \\
\hline \multicolumn{3}{|l|}{ Specialized behavioral health } \\
\hline Day treatment center & 300 & Fast Track agency record reviews \\
\hline Drug/alcohol clinic & 140 & Fast Track agency record reviews \\
\hline Foster home & 81 & Fast Track agency record reviews \\
\hline Group home & 147 & Fast Track agency record reviews \\
\hline In-home therapist & 105 & Fast Track agency record reviews \\
\hline Counselor/therapist & 90 & Fast Track agency record reviews \\
\hline Outpatient mental health center & 174 & Fast Track agency record reviews \\
\hline Psychiatric hospital & 406 & Fast Track agency record reviews \\
\hline Residential treatment center & 257 & Fast Track agency record reviews \\
\hline \multicolumn{3}{|l|}{ General health } \\
\hline Emergency department & 428 & Fast Track agency record reviews \\
\hline Family doctor & 85 & Fast Track agency record reviews \\
\hline General hospital & 526 & Fast Track agency record reviews \\
\hline \multicolumn{3}{|l|}{ Juvenile justice services } \\
\hline Arrest $^{a, b}$ & 1,705 & Cohen, Miller, \& Rossman ${ }^{34}$ \\
\hline Detention center & 108 & Fast Track agency record reviews \\
\hline \multicolumn{3}{|l|}{ School services } \\
\hline Grade retention $^{a, b}$ & 6,875 & National Center for Educational Statistics ${ }^{33}$ \\
\hline School counseling & 58 & Fast Track agency record reviews \\
\hline Special education $a, b$ & 6,141 & Forness \& Kavale ${ }^{32}$ \\
\hline Medications (behavioral) $)^{a, b}$ & 349 & Zuvekas $^{35}$ \\
\hline
\end{tabular}

